\title{
Linear Ultrasonic Array Development incorporating Cantor Set Fractal Geometry
}

\author{
H. Fang, Z. Qiu, R. L. O'Leary and A. Gachagan \\ Centre for Ultrasonic Engineering \\ University of Strathclyde \\ Glasgow, UK G1 1XW \\ Email: haoyu.fang@strath.ac.uk
}

\author{
A. J. Mulholland \\ Department of Mathematics and Statistics \\ University of Strathclyde \\ Glasgow, UK G1 1XH
}

\begin{abstract}
Naturally occurring resonating systems utilize structures containing a range of length scales to produce a broad operating bandwidth. It has previously been reported that a piezoelectric composite transducer based on a fractal geometry, which thereby introduces components with varying length scales, results in a wider operational bandwidth and a higher sensitivity. In this paper, the work is now extended to an ultrasonic array device using a Cantor Set (CS) fractal geometry. The behavior of this fractal array is explored using both finite element (FE) modeling and experimentation, including comparison with a conventional 2-2 linear array. The FE simulated pulse-echo responses correlate well with the experimental data, which indicates that the CS fractal array elements possessed a wider - $6 \mathrm{~dB}$ bandwidth $(57.3 \%$ against $49.4 \%)$, and a higher sensitivity, (11.4 mV against $8.9 \mathrm{mV}$ peakto-peak voltage) compared with a conventional 2-2 design. In addition, an improved crosstalk reduction is achieved by the $\mathrm{CS}$ fractal array. Images of a wire-water phantom produced by the two arrays using the total focusing method (TFM) and full matrix capturing (FMC) data shows that the CS fractal array outperforms the conventional 2-2 array in terms of image resolution and signal strength. Finally, another advanced fractal geometry comprising orthogonal CS fractal geometries, known as the Cantor Tartan (CT) is investigated to further enhance the bandwidth performance of the array, where a $-6 \mathrm{~dB}$ pulse-echo bandwidth of $68.1 \%$ can be predicted using FE modeling.
\end{abstract}

Keywords-Fractal Ultrasonic Array; Array Characterization; Wide Bandwidth;

\section{INTRODUCTION}

A typical ultrasonic array comprises a number of individual piezoelectric elements with the center-to-center spacing less than $\lambda \backslash 2$ [1]. Each individual element can be excited individually, by application of different time delays, termed as the focal law. Hence beam steering or focusing can be created electronically, improving the flexibility of the imaging process [2]. Ultrasound array techniques are extensively used in modern clinical diagnostic and therapeutic applications [3], such as real-time blood flow measurement [4] and non-invasive surgery [5] and in industrial applications such as non-destructive testing and evaluation (NDT\&E) [6] due to the advantage of high sensitivity, flexibility and its ability to be guided and focused easily [7].

The behavior of an ultrasonic array transducer can be modified by various schemes. Some researchers use materials with enhanced piezoelectric properties to improve the performance of the ultrasound transducer. For example, Wong et al. [8] designed a high frequency, $20 \mathrm{MHz}$, phased array ultrasound transducer with a PMN-PT single crystal material for high-resolution imaging. This single crystal material exhibited a wider operational bandwidth, when compared with other piezoceramic designs. The performance of an ultrasonic array can also be tailored by employing some electronic tuning component. Dziewierz et al. [9] developed an in-probe electronics printed circuit board (PCB) in order to provide electrical impedance matching between each array element and the associated drive electronics. The experimental results showed that a significant sensitivity and bandwidth enhancement can be realized after using such inprobe electrical matching. Moreover, the performance of the ultrasonic array can be enhanced via optimizing the structure of the active layer in the design. Yang et al. [10] developed a pseudo-random composite transducer by dicing the ceramic plate with two sets of cross cuts at different angles relative to the horizontal. The pulse-echo response bandwidth of this pseudo-random composite was increased when compared to an equivalent standard 1-3 composite design.

It has been reported in the authors' previous work that in a single element piezoelectric transducer design, using the Sierpinski Gasket (SG) fractal geometry to introduce elements with varying length scales can result in a wider operational bandwidth and higher sensitivity [11].

The approach is extended in this paper to investigate an ultrasonic linear array design using fractal geometry. In this work, a Cantor Set (CS) fractal geometry, shown in Fig. 1, at generation level II is employed to design a 2-2 connectivity piezoelectric composite based linear array with 24 elements. The design space of this CS fractal array is explored using finite element (FE) modeling (PZFlex, OnScale Inc, Cupertino, CA) and compared with a standard 2-2 connectivity, 24-element array device. The experimental performance of the fabricated array elements, both CS and conventional 2-2 array, are characterized and compared. The crosstalk performance of both array devices is measured using a 3D laser doppler vibrometer. The imaging and defect sizing capability of the two arrays is then evaluated by full matrix capture (FMC) and the total focusing method (TFM) with a wire-water phantom. Moreover, in order to introduce a higher fractal generation level configuration, an advanced fractal array with an orthogonal CS fractal geometry, called the Cantor Tartan (CT), is proposed and simulated using FE modeling.

The core funding for Mr Fang's studentship has been provided by the University of Strathclyde and the work has been carried out under the auspices of the UK Research Centre for NDE (EPSRC Grant EP/L022125/1). 


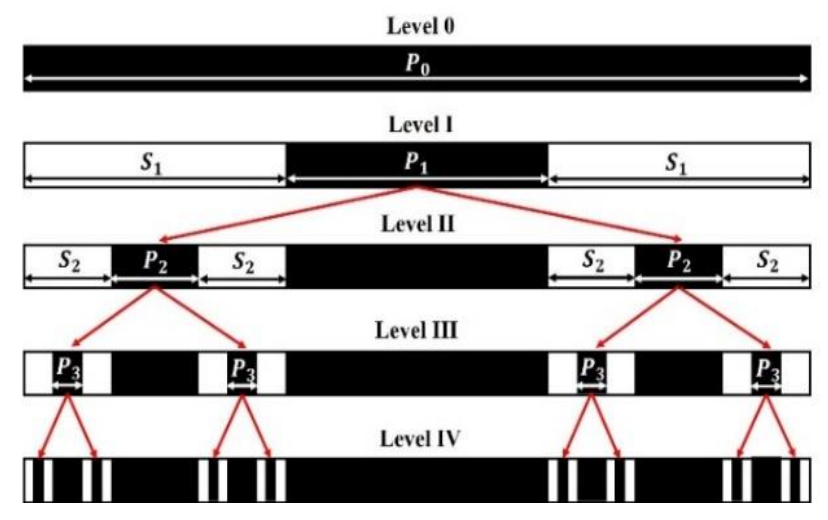

Fig. 1: Schematic diagram of the first four fractal generation levels of the CS fractal geometry (Black: Ceramic Pillar; White: Polymer Filler).

The widths $P_{n}$ and $S_{n}$ shown in Fig. 1 can be calculated via a iteration rule described in Equation (1) and (2) with a scaling factor, $k$ (where $0<k<0.5$ ).

$$
\begin{gathered}
P_{n}=P_{n-1} \cdot k \\
S_{n}=P_{0} \cdot k^{n},(n \geq 1)
\end{gathered}
$$

\section{FINITE ELEMENT MODELLING}

\section{A. Array Single Element Design}

FE models of one single element of a CS fractal array at fractal generation Level II and a conventional 2-2 linear array were built as shown in Fig. 2 (a) and (b), respectively. Both array designs have a thickness of $1.5 \mathrm{~mm}$ in the $\mathrm{z}$-dimension and an element length of $15 \mathrm{~mm}$ in the y-dimension. The kerf width is $0.13 \mathrm{~mm}$ and $0.15 \mathrm{~mm}$ for the CS fractal array and conventional array, respectively. However, it can be seen from Fig. 2 (a), that each individual CS fractal array element contains ceramic pillars in two different sizes $\left(\mathrm{P}_{1}=0.47 \mathrm{~mm}\right.$ and $\mathrm{P}_{2}=0.15 \mathrm{~mm}$ ) in the $\mathrm{x}$-dimension, where the pillar width of the conventional array element, $P_{c}$, is $0.15 \mathrm{~mm}$. The materials selected for the active and passive phases were PZT5H ceramic (Meggitt A/S, Kvistgard, Denmark) and CY1300/HY1301 (Robnor Resin Ltd, UK), respectively.

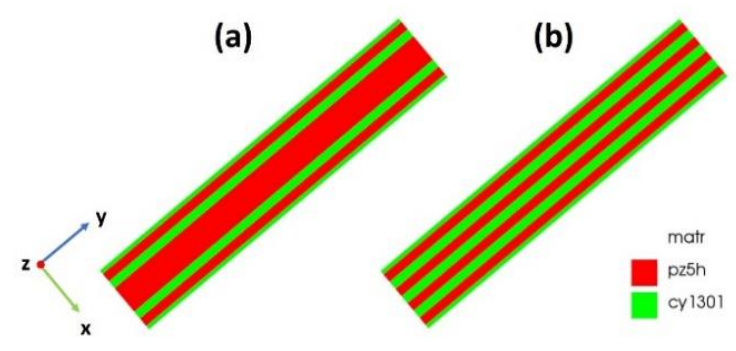

Fig. 2: FE model of a single element within the array, (a) Level II CS fractal; (b) 2-2 piezocomposite

\section{1) Electrical Impedance Profile}

The electrical impedance magnitude spectra of an element without matching and backing in each array design was simulated and is shown in Fig. 3. It can be seen that there are two resonant modes exhibited in the CS fractal array element, which are located at $817 \mathrm{kHz}$ and $1086 \mathrm{kHz}$. For the conventional array design, the resonant frequency of the element occurs at $1040 \mathrm{kHz}$.

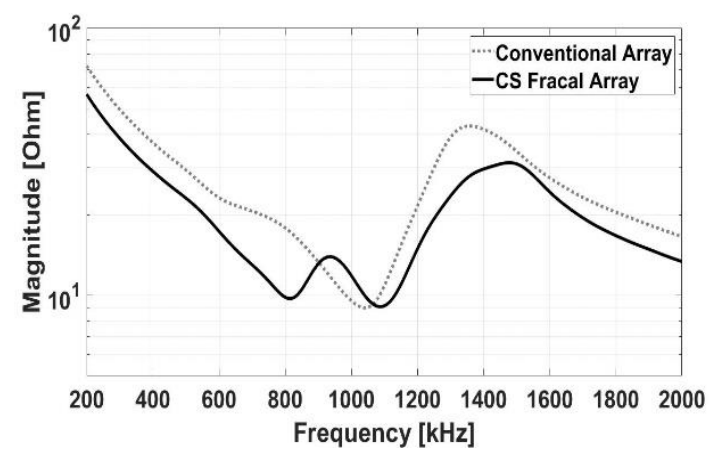

Fig. 3: FE derived electrical impedance magnitude spectrum of the CS and conventional array element

\section{2) Modal Analysis}

Following the electrical impedance analysis of the CS and conventional array element, the displacement mode shape at each resonant frequency in the thickness direction, $\mathrm{z}$ direction, of one array element from each array configuration was investigated and these are shown in Fig. 4 (a), (b) and (c). It can be seen from Fig. 4 (a), at the first resonant frequency of the CS fractal array element, $817 \mathrm{kHz}$, characteristics of a thickness mode vibration in the $\mathrm{z}$ direction can be seen in the pillar in the first fractal generation level. As shown in Fig. 4 (b), at the second resonant frequency of the CS fractal array element, $1086 \mathrm{kHz}$, a strong thickness vibration behavior can be seen in both the first and second fractal generation level pillars. Consequently, $1086 \mathrm{kHz}$ is considered to be the main thickness resonance frequency of the CS fractal array element. For the conventional array design in Fig. 4 (c), at its only resonant frequency, $1040 \mathrm{kHz}$, all the ceramic pillars vibrate uniformly in phase along the thickness direction.

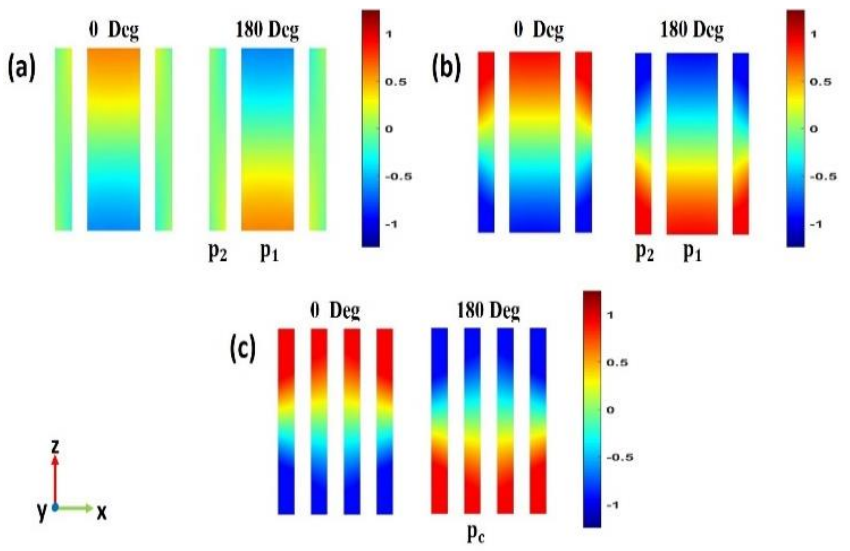

Fig. 4: Array element thickness displacement mode shape in $\mathrm{z}$ direction of (a) CS fractal array at $817 \mathrm{kHz}$; (b) CS fractal array at $1086 \mathrm{kHz}$ and (c) conventional array at $1040 \mathrm{kHz}$

\section{B. Pulse-Echo Modeling of Array Element}

In order to assess the impulse response of each design, both array designs were configured with a dual matching layer, where the acoustic impedance of the inner and outer matching layer is 5.72 MRayls and 2.78 MRayls, respectively. A backing of 8.62 MRayls was selected to be manufactured from tungsten loaded epoxy.

The pulse-echo response of an array element was simulated in a water load using PZFlex for both array designs. The results are shown in Fig. 5 (a) and (b) and Table I. 
(a)
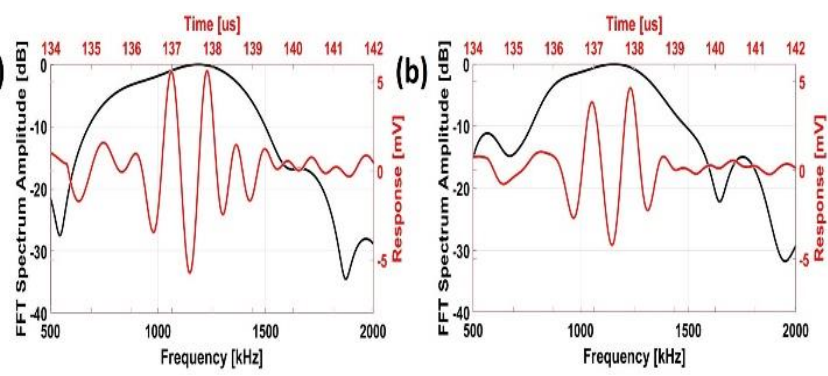

Fig. 5: Simulated pulse-echo time-domain waveform (red) and frequency spectrum (black) of: (a) CS fractal array (b) conventional linear array

Table I: Pulse-echo modeling result summary

\begin{tabular}{|c|c|c|}
\hline & $\boldsymbol{V}_{\boldsymbol{p}-\boldsymbol{p}}$ & 6 dB Bandwidth \\
\hline CS Fractal Array & $11.4 \mathrm{mV}$ & $57.3 \%$ \\
\hline Conventional Array & $8.9 \mathrm{mV}$ & $49.4 \%$ \\
\hline
\end{tabular}

From Fig. 5 and Table I, a $16.0 \%$ bandwidth improvement has been achieved by the CS fractal array element, with respect to the conventional 2-2 array element. As for the sensitivity, the peak-to-peak received voltage $\left(V_{p-p}\right)$, of the CS fractal array element is $28 \%$ higher than the conventional array element.

\section{EXPERIMENTAL VALIDATION}

\section{A. Array Manufacturing}

The piezocomposite active layer of the CS fractal array and the conventional 2-2 array were fabricated using the conventional dice-and-fill technique. The scratch dicing technique was utilised for element electrical isolation, where 24 linear elements were defined for each array device and a flexible printed circuit board (PCB) was employed for electrical interconnection (see Fig. 6 (a)). In Fig. 6 (b), both array designs were backed and matched to a water load via a dual matching layer scheme as detailed in the Section II (B). (a)

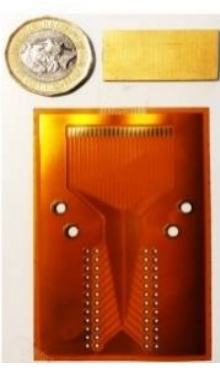

(b)

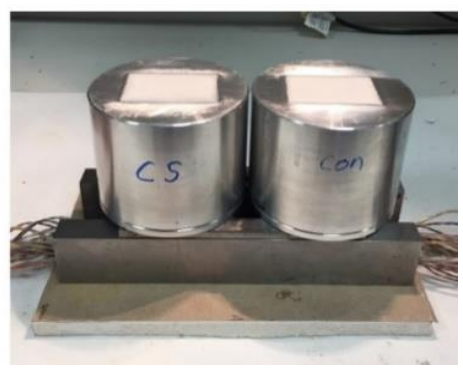

Fig. 6: (a) Piezocomposite array active layer and Flexible PCB (b) Fabricated CS Fractal and conventional linear array

\section{B. Array surface Dilation}

The array element surface dilation of the fabricated CS fractal array and conventional linear array was measured using a Polytec PSV-300 scanning laser Doppler vibrometer (Polytec Inc.) and the result is shown in Fig. 7. The center positions of the driven element and the two adjacent elements at either side of the driven element are marked with vertical red dash lines in Fig. 7.

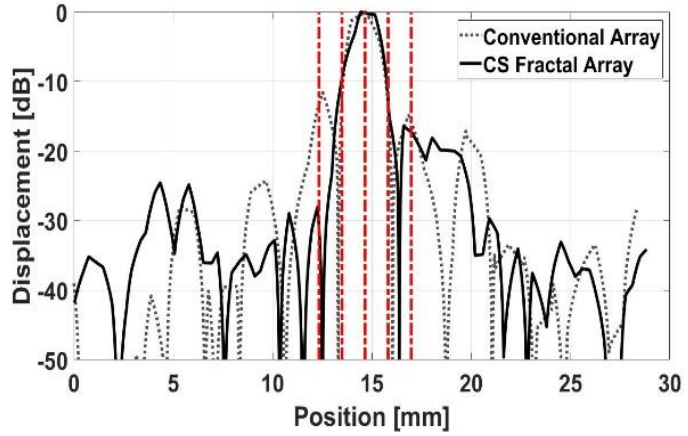

Fig. 7: Measured crosstalk of CS fractal and conventional array

It can be seen from Fig. 7 that the crosstalk of the CS fractal array is at a similar level to the conventional array for the first adjacent elements but 5 to $15 \mathrm{~dB}$ lower for the second adjacent elements. This is because that the CS fractal array has a nonuniform periodicity, which leads to an attenuation for the Lamb wave propagating across the array aperture [12], [13].

\section{Pulse-Echo Measurement}

The pulse echo responses for each element of both array devices were measured using a multi-element array controller, FIToolbox (Diagnostic Sonar Ltd, UK), and compared with the FE simulated results, as shown in Fig. 8 (a) and (b).
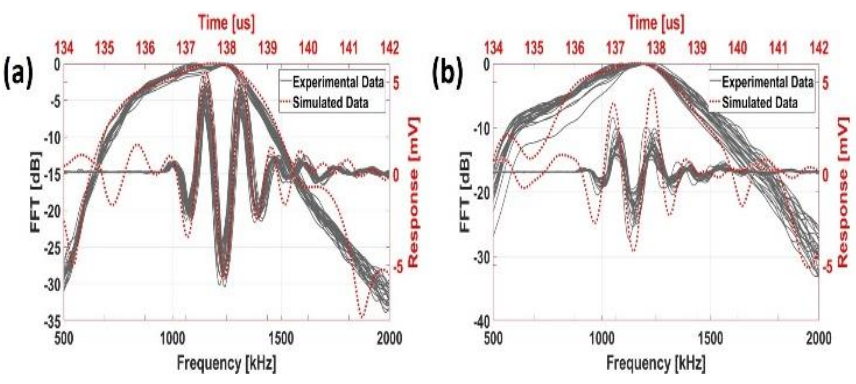

Fig. 8: Measured and simulated pulse-echo time-domain waveform and frequency spectrum (a) CS fractal array (b) conventional array

It can be seen in Fig. 8 that all of the elements behaved uniformly in each array and the experimental results correlated well with the simulation data.

\section{Array Imaging Performance}

A copper wire-water phantom as shown in Fig. 9 was built to evaluate and compare the imaging and defect sizing capability of both arrays, where the diameter of the wires are $0.72 \mathrm{~mm}(\sim 0.5 \lambda), 1.12 \mathrm{~mm}(\sim 0.75 \lambda)$ and $1.60 \mathrm{~mm}(\sim 1.0$ $\lambda)$, respectively. The resultant FMC/TFM images [14] produced by both arrays is shown in Fig. 10. (a)

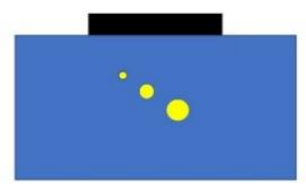

(b)

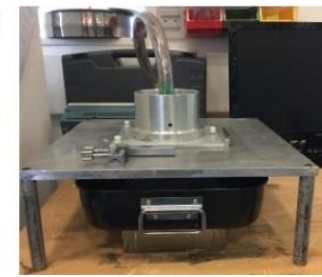

Fig. 9: (a) Imaging phantom schematic; (b) FMC/TFM experimental setup 

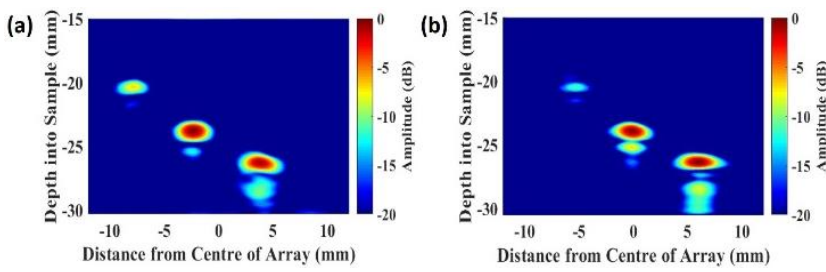

Fig. 10: TFM imaging result: (a) CS fractal array; (b) conventional array

It can be seen that the CS array produced a higher resolution TFM image compared to the conventional array and importantly, the imaging artefacts associated with each wire indication are reduced for the CS array. Using the $-6 \mathrm{~dB}$ sizing method, the accuracy of the CS fractal array for the wire with $0.5 \lambda, 0.75 \lambda$ and $1.0 \lambda$ diameter is $4.4 \%, 20.5 \%$ and $47.7 \%$ better than the conventional linear array. Moreover, the signal strength in the CS array image is $3.8 \mathrm{~dB}$ higher than that provided by the conventional array for the wire with $0.5 \lambda$ diameter.

\section{Advanced Cantor Tartan (CT) Fractal Array CONFIGURATION}

In order to introduce a higher fractal generation level configuration, an advanced CT fractal array element was designed with orthogonal fractal geometries, which is shown in Fig. 11 (a). This CT array element is a combination of a Level II CS fractal geometry in azimuth and a Level IV CS fractal geometry in elevation. The pulse-echo response of this CT fractal array element was simulated in PZFlex and the result is shown in Fig. 11 (b).
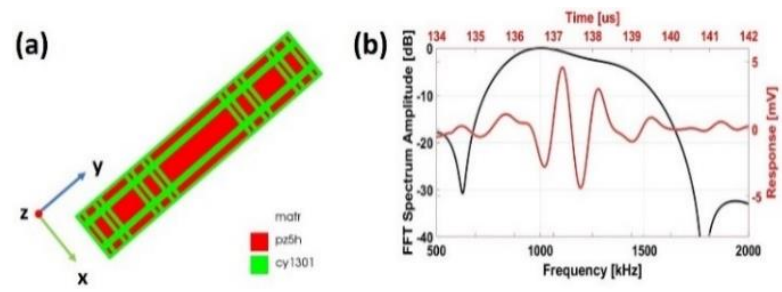

Fig. 11: (a) Advanced orthogonal CT fractal array element; (b) FE simulated pulse-echo response of CT fractal array element

It can be seen in Fig. 11 (b) that the CT fractal array element has a $-6 \mathrm{~dB}$ bandwidth of $68.1 \%$ and a peak-to-peak received voltage, $V_{p-p}$, of $9.0 \mathrm{mV}$. When compared to the CS fractal and conventional linear array element, see Table I, this CT fractal design has the best bandwidth performance, as a result of the introduction of a high fractal generation level. The CT simulation results indicate that the sensitivity is lower than the CS configuration, but comparable with the conventional 2-2 design.

\section{CONCLUSION \& FUTURE WORK}

This work describes the implementation of using a Cantor Set fractal geometry as the structure of a linear ultrasonic array design. FE models of a CS fractal array, a conventional linear array and an advanced orthogonal Cantor Tartan (CT) fractal array element have been investigated. Good bandwidth and sensitivity performance was demonstrated in the fractal array designs, when compared to the conventional array configurations, especially for the CT array element comprising a more complex fractal geometry.

A CS fractal and a conventional linear array with 24 elements were fabricated and characterised. The pulse-echo response of all elements behaved uniformly in each array design and the experimental data matched well with the FE simulated results. The crosstalk of both array devices was measured, where a 5 to $15 \mathrm{~dB}$ crosstalk reduction for the second adjacent elements can be achieved by the CS fractal array design. Lastly, when compared to the conventional array, an improved imaging capability in terms of the image resolution and signal strength can be obtained by the CS fractal array.

In the future, the CT fractal array will be fabricated and fully characterised regarding its pulse-echo response, crosstalk and imaging performance.

\section{REFERENCES}

[1] B. W. Drinkwater and P. D. Wilcox, "Ultrasonic arrays for nondestructive evaluation: A review," NDT E Int., vol. 39, no. 7, pp. 525541, 2006.

[2] A. McNab and M. J. Campbell, "Ultrasonic phased arrays for nondestructive testing," NDT Int., vol. 20, no. 6, pp. 333-337, 1987.

[3] O. T. Von Ramm and S. W. Smith, "Beam Steering with Linear Arrays," IEEE Trans. Biomed. Eng., vol. BME-30, no. 8, pp. 438-452, 1983.

[4] L. Zhang, X. Xu, C. Hu, L. Sun, J. T. Yen, J. M. Cannata, and K. K. Shung, "A high-frequency, high frame rate duplex ultrasound linear array imaging system for small animal imaging," Ultrason. Ferroelectr. Freq. Control. IEEE Trans., vol. 57, no. 7, pp. 1548-1557, 2010.

[5] E. B. Hutchinson, "Intracavitary ultrasound phased arrays for noninvasive prostate surgery," IEEE Trans. Ultrason. Ferroelectr. Freq. Control, vol. 43, no. 6, pp. 1032-1042, 1996.

[6] S. Mahaut, O. Roy, C. Beroni, and B. Rotter, "Development of phased array techniques to improve characterisation of defect located in a component of complex geometry," Ultrasonics, vol. 40, no. 1-8, pp. 165-169, 2002.

[7] M. Li and G. Hayward, "Ultrasound nondestructive evaluation (NDE) imaging with transducer arrays and adaptive processing," Sensors, vol. 12 , no. 1 , pp. 42-54, 2012

[8] C. M. Wong, Y. Chen, H. Luo, J. Dai, K. H. Lam, and H. L. wa Chan, "Development of a $20-\mathrm{MHz}$ wide-bandwidth PMN-PT single crystal phased-array ultrasound transducer," Ultrasonics, vol. 73, pp. 181-186, 2017.

[9] J. Dziewierz and A. Gachagan, "Enhancing 2D phased array sensitivity and bandwidth using in-probe electronics," IEEE Int. Ultrason. Symp. IUS, pp. 2400-2403, 2012.

[10] H. C. Yang, J. Cannata, J. Williams, and K. Shung, "Crosstalk reduction for high-frequency linear-array ultrasound transducers using 1-3 piezocomposites with pseudo-random pillars," IEEE Trans. Ultrason. Ferroelectr. Freq. Control, vol. 59, no. 10, pp. 2312-2321, 2012.

[11] H. Fang, Z. Qiu, R. L. O’Leary, A. Gachagan, and A. J. Mulholland, "Improving the operational bandwidth of a 1-3 piezoelectric composite transducer using Sierpinski Gasket fractal geometry," IEEE Int. Ultrason. Symp. IUS, vol. 2016-Novem, 2016.

[12] G. Hayward and J. Hyslop, "Determination of lamb wave dispersion data in lossy anisotropic plates using time domain finite element analysis. Part I: theory and experimental verification," IEEE Trans. Ultrason. Ferroelectr. Freq. Control, vol. 53, no. 2, pp. 443-448, Feb. 2006.

[13] G. Hayward and J. Hyslop, "Determination of Lamb wave dispersion data in lossy anisotropic plates using time domain finite element analysis. Part II: Application to 2-2 and 1-3 piezoelectric composite transducer arrays," IEEE Trans. Ultrason. Ferroelectr. Freq. Control, vol. 53, no. 2, pp. 449-455, 2006.

[14] C. Holmes, B. W. Drinkwater, and P. D. Wilcox, "Post-processing of the full matrix of ultrasonic transmit-receive array data for nondestructive evaluation," NDT E Int., vol. 38, no. 8, pp. 701-711, Dec. 2005 . 